\title{
Autotransfusion Procedure
}

National Cancer Institute

\section{Source}

National Cancer Institute. Autotransfusion Procedure. NCI Thesaurus. Code C50870.

Infusion of previously obtained autologous blood or blood products back to the same individual. 